\title{
An Alternate Method for Computation of Transfer Function Matrix
}

\author{
Appukuttan K. K. and Suma Bhat \\ Department of Mechanical Engineering, National Institute of Technology, Karnataka 575025, India \\ Correspondence should be addressed to Suma Bhat, sumaudayabhat@yahoo.com
}

Received 24 September 2010; Accepted 22 December 2010

Academic Editor: Ian R. Petersen

Copyright ( 2010 Appukuttan K. K. and S. Bhat. This is an open access article distributed under the Creative Commons Attribution License, which permits unrestricted use, distribution, and reproduction in any medium, provided the original work is properly cited.

A direct and simple numerical method is presented for calculating the transfer function matrix of a linear time invariant multivariable system $(A, B, C)$. The method is based on the matrix-determinant identity, and it involves operations with an auxiliary vector on the matrices. The method is computationally faster compared to Liverrier and Danilevsky methods.

\section{Introduction}

In the design of multivariable control systems by frequency domain methods, the computation of the transfer function matrix from the state space description of the system plays an important role. The computation based on Liverrier method [1] is regarded as unsatisfactory especially when the system order is high or its eigenvalues are widely distributed. Patel [2] has presented a method whereby each numerator element of the transfer function matrix is calculated as the difference between the characteristic polynomials of two matrices which are related to the system state matrix, and the polynomials are computed by the method of Danilevsky [1]. Danilevsky method is used in many numerically intensive calculations where extreme eigenvalues have to be estimated [3-5]. Daly [6] has given a method for the transfer function matrix evaluation which involves a sequence of similarity transformations on the Rosenbrock system matrix [7] and resembles Danilevsky technique.

This paper, instead of using the Danilevsky method, presents a direct procedure to compute the characteristic polynomials associated with each numerator element of the transfer function matrix. The method makes use of an auxiliary output vector and the corresponding single-output system is transformed to its row companion form to give the coefficients of the characteristic polynomial. The above transformation is carried out by the algorithm given by Hickin and Sinha [8] which is based on the transformation of a matrix to its Hermite normal form.

\section{Transfer Function Matrix}

Consider the $n$ th-order, $m$ inputs, $\ell$ outputs, linear-time invariant system described by

$$
\begin{gathered}
\dot{x}=A x+B u, \\
y=C x,
\end{gathered}
$$

where $A, B$, and $C$ are matrices of appropriate dimensions. The $\ell \times m$ transfer function matrix of the system is given by

$$
G(s)=C(s I-A)^{-1} B=\frac{W(s)}{F(s)},
$$

where $W(s)=C \operatorname{adj}(s I-A) B$ is the $\ell \times m$ numerator transfer function matrix and $F(s)=\operatorname{det}(s I-A)=s^{n}+f_{1} s^{n-1} \cdots+f_{n}$ is the characteristic polynomial. Any typical element $W_{i j}(s)$ of $W(s)$ is

$$
W_{i j}(s)=c_{i} \operatorname{adj}(s I-A) b_{j}, \quad i=1, \ldots, \ell, j=1, \ldots, m .
$$

Using the matrix-determinant identity [9]

$$
\operatorname{Det}(R+e d)=\operatorname{det}(R)+d \operatorname{adj}(R) e,
$$


where $R$ is an $n \times n$ matrix and $e$ and $d$ are $n \times 1$ and $1 \times n$ vectors, respectively. $W_{i j}(s)$ can be expressed as:

$$
W_{i j}(s)=\operatorname{det}\left(s I-A+b_{j} c_{i}\right)-\operatorname{det}(s I-A) .
$$

Patel [2] has suggested the method of Danilevsky for computing the polynomials in (5). A simple and direct method to compute the coefficients of the polynomials is given below.

\section{Characteristic Polynomial of $A$}

For a given matrix A, let $r$ represent $1 \times n$ auxiliary output vector such that $(A, r)$ is observable. Form the matrix:

$$
R=\left[\begin{array}{c}
r \\
r A \\
r A^{2} \\
\vdots \\
r A^{n}
\end{array}\right]=\left[\begin{array}{c}
r_{1} \\
r_{2} \\
\vdots \\
r_{n} \\
r_{n+1}
\end{array}\right] .
$$

For the above single-output system, the transformation of $R$ to its row companion form will result in a canonical form where the first $n$ rows will be unit vectors appearing in their natural order and the elements of the last row will be the coefficients of the characteristic polynomial of $A$. Thus, $\operatorname{det}(s I-A)$ can be obtained directly from the row companion form representation of $R$.

The transformation of the matrix $R$ to its row companion form is done in $\mathrm{n}$ steps on a row basis starting from the first row. For each row select the element $r_{i i}$ as pivot and calculate

$$
\begin{array}{r}
\bar{r}_{i}=\left(r_{i}-e_{i}\right), \quad R_{i}=R_{i-1}-\left(r_{i i}\right)^{-1}\left(R_{i-1}\right) e_{i}^{T} \bar{r}_{i}, \\
i=1,2, \ldots, n, R_{0}=R,
\end{array}
$$

where $e_{i}$ is a unit row vector. Each step of transformation will result in the elements of the row $r_{i}$ being expressed as the unit vector $e_{i}$.

After the $n$ step transformation, the matrix $R_{n}$ will be in the Hermite normal form where the unit row vectors appear in their natural order in the first $n$ rows and the elements of the last row will be $\left(-f_{n},-f_{n-1}, \ldots,-f_{1}\right)$. Thus, the coefficients of the characteristic polynomial $F(s)$ of $A$ are obtained.

The polynomial of degree $(n-1)$ corresponding to the element $w_{i j}(s)$ is obtained as the difference between the characteristic polynomials of $\left(A-b_{j} c_{i}\right)$ and $A$. Thus in all $(\ell m+1)$, characteristic polynomials have to be computed to evaluate the complete transfer function matrix $G(s)$.

\section{Numerical Example One}

$$
A=\left[\begin{array}{ccc}
0 & -2 & 1 \\
1 & 0 & 0 \\
0 & 0 & 0
\end{array}\right], \quad B=\left[\begin{array}{ll}
1 & 0 \\
0 & 0 \\
0 & 1
\end{array}\right], \quad C=\left[\begin{array}{ccc}
2 & 0 & 0 \\
0 & -2 & 2
\end{array}\right] .
$$

4.1. Computation of $\operatorname{det}(s I-A)$. Let $r=\left(\begin{array}{lll}1 & 1 & 0\end{array}\right)$ as per $(6)$ :

$$
R=\left[\begin{array}{ccc}
1 & 1 & 0 \\
1 & -2 & 1 \\
-2 & -2 & 1 \\
-2 & 4 & -2
\end{array}\right]
$$

The transformation with (7) gives

$$
\begin{aligned}
& R_{1}=R-\left[\begin{array}{c}
1 \\
1 \\
-2 \\
-2
\end{array}\right]\left[\begin{array}{lll}
0 & 1 & 0
\end{array}\right]=\left[\begin{array}{ccc}
1 & 0 & 0 \\
1 & -3 & 1 \\
-2 & 0 & 1 \\
-2 & 6 & -2
\end{array}\right], \\
& R_{2}=R_{1}-\left[\begin{array}{c}
0 \\
-1 \\
0 \\
2
\end{array}\right]\left[\begin{array}{lll}
1 & -4 & 1
\end{array}\right]=\left[\begin{array}{ccc}
1 & 0 & 0 \\
0 & 1 & 0 \\
-2 & 0 & 1 \\
0 & -2 & 0
\end{array}\right], \\
& R_{3}=R_{2}-\left[\begin{array}{l}
0 \\
0 \\
1 \\
0
\end{array}\right]\left[\begin{array}{lll}
-2 & 0 & 0
\end{array}\right]=\left[\begin{array}{ccc}
1 & 0 & 0 \\
0 & 1 & 0 \\
0 & 0 & 1 \\
0 & -2 & 0
\end{array}\right] .
\end{aligned}
$$

Hence $\operatorname{det}(s I-A)=s^{3}+2 s$.

4.2. To Find the Element $w_{11}(s)$ of $W(s)$. The characteristic polynomial of $\left(A-b_{1} c_{1}\right)$ is computed using (6) and (7) and is $\left(s^{3}+2 s^{2}+2 s\right)$. Hence the elements $w_{11}(s)$ as per (5) are $2 s^{2}$. Similarly all the other elements of $W(s)$ can be computed.

\section{Numerical Example Two}

The jet engine model which used NEC International form [10] is considered to compare the numerical properties of the present method with other known methods. The fifthorder model of the jet engine is considered for computing the coefficients of the characteristic polynomial. The state matrix is: 
TABLE 1: Comparison of computation of coefficients of characteristic polynomial.

\begin{tabular}{lccc}
\hline Coefficients & Liverrier & $\begin{array}{c}\text { Methods } \\
\text { Danilevsky }\end{array}$ & Present method \\
\hline$f_{1}$ & 22.716 & 200.716 & 200.716 \\
$f_{2}$ & 4794.685 & 4794.685 & 4794.685 \\
$f_{3}$ & 43338.55 & 43338.55 & 43338.55 \\
$f_{4}$ & 166117.00 & 166117.10 & 166117.10 \\
$f_{5}$ & 214851.50 & 214840.00 & 214840.00 \\
\hline
\end{tabular}

$$
A=\left[\begin{array}{ccccc}
-0.3245 E+01 & -0.2158 E+01 & -0.9155 E+03 & 0.5731 E+00 & 0.1342 E+03 \\
0.1642 E+01 & -0.5941 E+01 & -0.2816 E+01 & 0.1897 E+01 & 0.5705 E+00 \\
0.1685 E-01 & -0.2854 E-01 & -0.1003 E+01 & 0.7994 E-02 & 0.5887 E+01 \\
0.000 & 0.000 & 0.000 & 1.000 E+02 & 0.000 \\
-0.2613 E+01 & 0.6862 E+01 & 0.7405 E+03 & 0.1195 E+01 & -0.1715 E+01
\end{array}\right]
$$

The computation was done on FORTRAN program, and results are shown in Table 1 . In the above matrix $A$, the ratio between the largest and the smallest element is of the order $10^{5}$. The present method gives identical results as the Danilevsky method, whereas the Liverrier method shows a small difference in the coefficients values in the last two rows. The execution time for Liverrier, Danilevsky, and present methods was found to be $0.43,0.36$, and 0.14 seconds, respectively. The eigenvalues are $-174.653,-10$, $-6.671+j 1.293,-6.671-j 1.293$, and -2.629 . The eigenvalues computed by MATLAB are in agreement with the above results.

\section{Conclusions}

A simple and direct method of computing the transfer function matrix of the system $(A, B, C)$ has been presented. All the $(\ell m+1)$ polynomials of the transfer function matrix are computed from the characteristic polynomials of $(\ell m+1)$ matrices related to the system state matrix. The coefficients of the above polynomials have been evaluated by a simple transformation of an auxiliary single-output system to its row companion form. The transformation is easily programmed on the computer.

\section{References}

[1] D. K. Faddeev and V. N. Faddeeva, Computational Methods of Linear Algebra, Freeman, San Francisco, Calif, USA, 1963.

[2] R. V. Patel, "On the computation of numerator transfer functions of linear systems," IEEE Transactions on Automatic Control, vol. 18, no. 4, pp. 400-401, 1973.

[3] S. L. Liu and S. Y. Wang, "Sensitivity analysis of nonnegative irreducible matrices," Applied Mathematics Letters, vol. 12, no. 2, pp. 121-124, 1999.
[4] H. R. Dullin and A. V. Tsygvintsev, "On the analytical integrity of the rattleback problem," Annales de la Faculte des Sciences de Toulouse Ser, vol. 6, no. 17, pp. 495-517, 2008.

[5] M. Nalbant, H. Gökkaya, I. Toktaş, and G. Sur, "The experimental investigation of the effects of uncoated, PVD- and CVD-coated cemented carbide inserts and cutting parameters on surface roughness in CNC turning and its prediction using artificial neural networks," Robotics and Computer-Integrated Manufacturing, vol. 25, no. 1, pp. 211-223, 2009.

[6] K. C. Daly, "A Computational procedure for transfer function evaluation," International Journal of Control, vol. 20, no. 4, pp. 569-576, 1974.

[7] H. H. Rosenbrock, State Space and Multivariable Theory, Nelson, Ontario, Canada, 1970.

[8] J. Hickin and N. K. Sinha, "On the transformation of linear multivariable systems to canonical forms," International Journal of Systems Science, vol. 10, no. 7, pp. 783-796, 1979.

[9] I. W. Sandberg, "On the theory of linear multi-loop feedback systems," Bell System Technical Journal, vol. 42, pp. 355-382, 1963.

[10] R. J. Miller and R. D. Hackney, F16 Multivariable Control System Models/Design Criteria, Fla, USA, Pratt and Whitney Aircraft Group, United Technologies Corporation, 1976. 

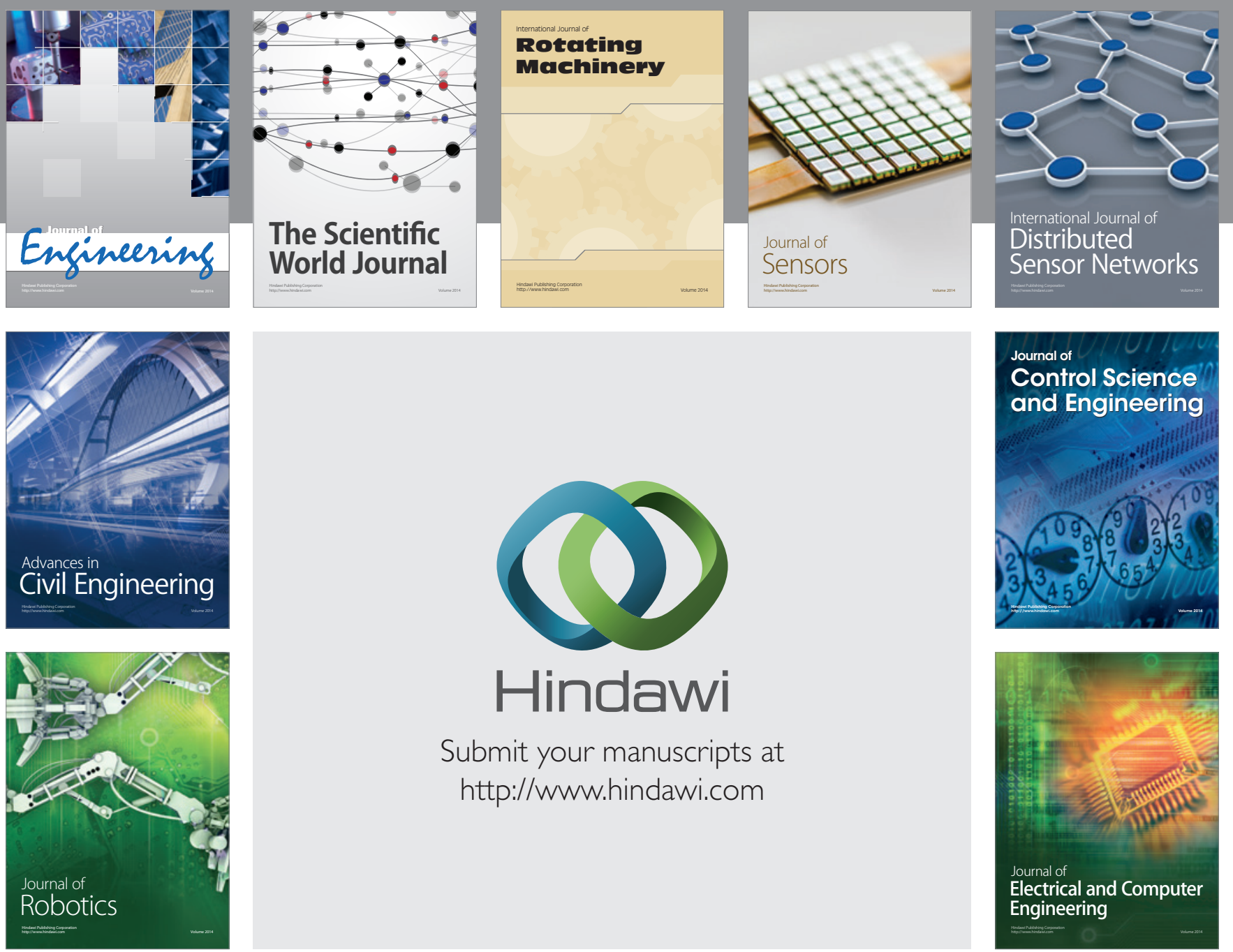

Submit your manuscripts at

http://www.hindawi.com
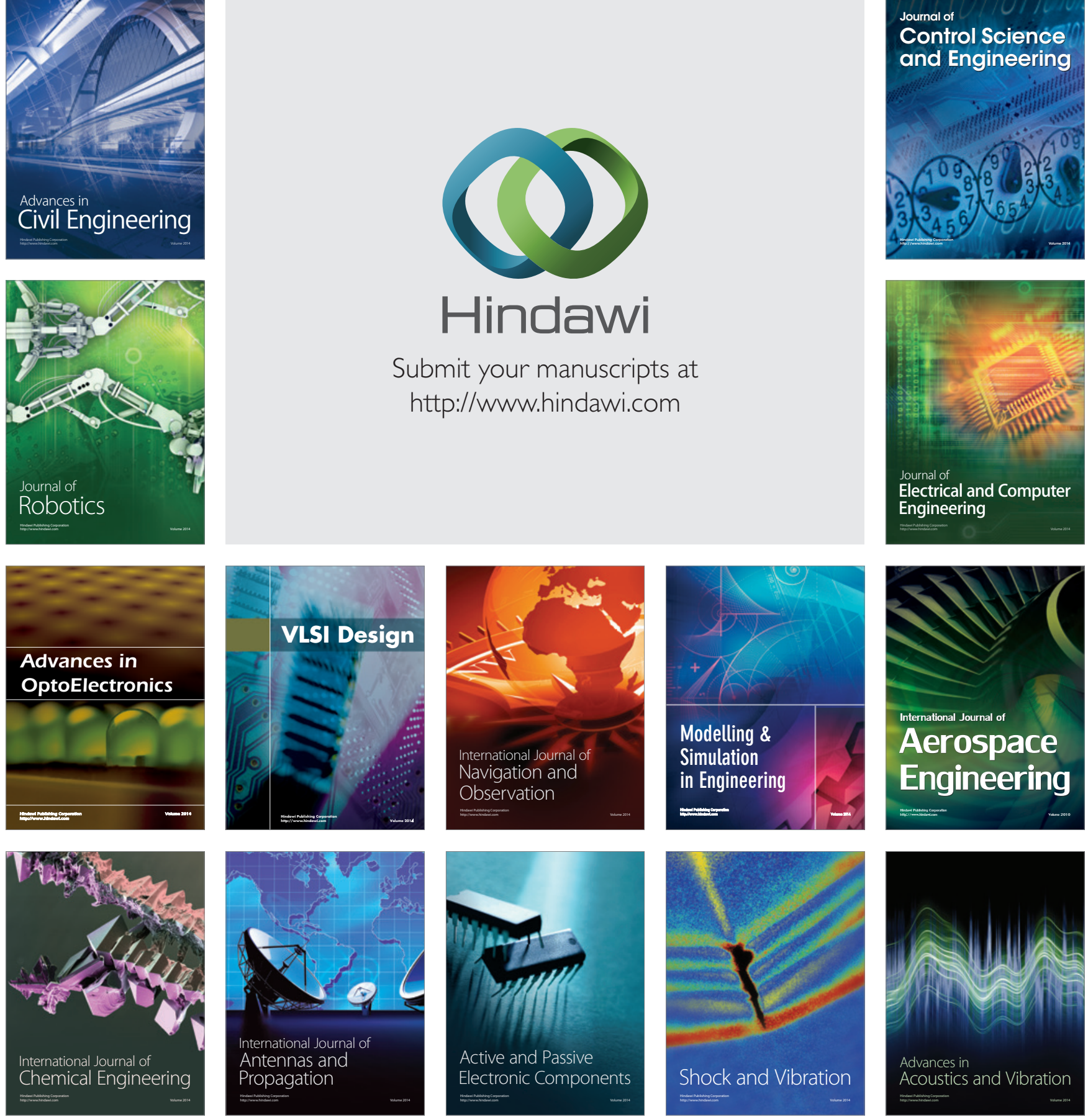